\title{
Discovery of $\boldsymbol{\gamma}$-lactam alkaloids derivatives as potential fungicidal agents targeting steroid biosynthesis
}

\author{
Daye Huang, ${ }^{\dagger}$ Shuangshuang Wang, ${ }^{\ddagger}$ Di Song, ${ }^{\ddagger}$ Xiufang Cao $, *, \ddagger$ Wenbo Huang, ${ }^{\dagger}$ Shaoyong Ke ${ }^{*} \dagger$ \\ †National Biopesticide Engineering Research Centre, Hubei Biopesticide Engineering Research Centre, \\ Hubei Academy of Agricultural Science, Wuhan 430064, China \\ Department of Chemistry, College of Science, Huazhong Agricultural University, Wuhan 430070, \\ China
}

\section{Supporting Information}

\section{Experimental}

\section{Chemistry}

All starting materials and reagents were obtained from commercial suppliers and used without further purification unless otherwise specified. NMR spectra were recorded on a Brucker spectrometer at 600 $\mathrm{MHz}$ with the $\mathrm{CDCl}_{3}$ as the solvent and TMS as the internal standard. Mass spectra were performed on a Waters ACQUITY UPLC ${ }^{\circledR}$ H-CLASS PDA (Waters $\left.{ }^{\circledR}\right)$ instrument. Optical rotations were measured on an Olympus CX21 (Tokyo, Japan), SEM images were visualized and obtained using Hitachi SU8100 (Japan), and TEM images were obtained using Hitachi HT7700 (Japan). Analytical thin-layer chromatography was carried out on precoated plates, and spots were visualized with ultraviolet light.

\section{Plant pathogenic fungi}

P. capsici, P. aphanidermatum, R. solani, B. cinerea, A. solani, P. theae, C. cassiicola, and F. oxysporum were obtained from Hubei Biopesticide Engineering Research Centre. The strains were retrieved from the storage tube and incubated in PDA at $25^{\circ} \mathrm{C}$ for a week to get new mycelia for the antifungal assay.

\section{Label-free quantitative proteomics analysis}

Protein Extraction

Compound D1 $(10 \mu \mathrm{g} / \mathrm{mL})$ toward $P$. capsici was used for the label-free quantitative proteomics analysis. 
The sample was grinded by liquid nitrogen into cell powder and then transferred to a 5-mL centrifuge tube. After that, four volumes of lysis buffer ( $8 \mathrm{M}$ urea, 1\% Triton-100, $10 \mathrm{mM}$ dithiothreitol, and 1\% Protease Inhibitor Cocktail) was added to the cell powder, followed by sonication three times on ice using a high intensity ultrasonic processor (Scientz). (Note: For PTM experiments, inhibitors were also added to the lysis buffer, e.g. $3 \mu \mathrm{M}$ TSA and $50 \mathrm{mM}$ NAM for acetylation.) The remaining debris was removed by centrifugation at $20,000 \mathrm{~g}$ at $4{ }^{\circ} \mathrm{C}$ for $10 \mathrm{~min}$. Finally, the protein was precipitated with cold $20 \% \mathrm{TCA}$ for $2 \mathrm{~h}$ at $-20^{\circ} \mathrm{C}$. After centrifugation at $12,000 \mathrm{~g} 4{ }^{\circ} \mathrm{C}$ for $10 \mathrm{~min}$, the supernatant was discarded. The remaining precipitate was washed with cold acetone for three times. The protein was re-dissolved in $8 \mathrm{M}$ urea and the protein concentration was determined with BCA kit according to the manufacturer's instructions.

\section{Trypsin Digestion}

For digestion, the protein solution was reduced with $5 \mathrm{mM}$ dithiothreitol for $30 \mathrm{~min}$ at $56{ }^{\circ} \mathrm{C}$ and alkylated with $11 \mathrm{mM}$ iodoacetamide for $15 \mathrm{~min}$ at room temperature in darkness. The protein sample was then diluted by adding $100 \mathrm{mM}$ TEAB to urea concentration less than $2 \mathrm{M}$. Finally, trypsin was added at 1:50 trypsin-to-protein mass ratio for the first digestion overnight and 1:100 trypsin-to-protein mass ratio for a second 4 h-digestion.

\section{HPLC Fractionation}

The tryptic peptides were fractionated into fractions by high $\mathrm{pH}$ reverse-phase HPLC using Thermo Betasil C18 column (5 $\mu \mathrm{m}$ particles, $10 \mathrm{~mm}$ ID, $250 \mathrm{~mm}$ length). Briefly, peptides were first separated with a gradient of $8 \%$ to $32 \%$ acetonitrile (pH 9.0) over 60 min into 60 fractions. Then, the peptides were combined into 6 fractions and dried by vacuum centrifuging.

\section{LC-MS/MS Analysis}

The tryptic peptides were dissolved in $0.1 \%$ formic acid (solvent A), directly loaded onto a home-made reversed-phase analytical column (15-cm length, $75 \mu \mathrm{m}$ i.d.). The gradient was comprised of an increase from $6 \%$ to $23 \%$ solvent B ( $0.1 \%$ formic acid in $98 \%$ acetonitrile) over $26 \mathrm{~min}, 23 \%$ to $35 \%$ in $8 \mathrm{~min}$ and climbing to $80 \%$ in $3 \mathrm{~min}$ then holding at $80 \%$ for the last $3 \mathrm{~min}$, all at a constant flow rate of 400 $\mathrm{nL} / \mathrm{min}$ on an EASY-nLC 1000 UPLC system. 
The peptides were subjected to NSI source followed by tandem mass spectrometry (MS/MS) in Q ExactiveTM Plus (Thermo) coupled online to the UPLC. The electrospray voltage applied was $2.0 \mathrm{kV}$. The $\mathrm{m} / \mathrm{z}$ scan range was 350 to 1800 for full scan, and intact peptides were detected in the Orbitrap at a resolution of 70,000. Peptides were then selected for MS/MS using NCE setting as 28 and the fragments were detected in the Orbitrap at a resolution of 17,500. A data-dependent procedure that alternated between one MS scan followed by 20 MS/MS scans with 15.0s dynamic exclusion. Automatic gain control (AGC) was set at 5E4. Fixed first mass was set as $100 \mathrm{~m} / \mathrm{z}$.

\section{Database Search}

The resulting MS/MS data were processed using Maxquant search engine (v.1.5.2.8). Tandem mass spectra were searched against human uniprot database concatenated with reverse decoy database. Trypsin/P was specified as cleavage enzyme allowing up to 4 missing cleavages. The mass tolerance for precursor ions was set as $20 \mathrm{ppm}$ in First search and $5 \mathrm{ppm}$ in Main search, and the mass tolerance for fragment ions was set as 0.02 Da. Carbamidomethyl on Cys was specified as fixed modification and acetylation modification and oxidation on Met were specified as variable modifications. FDR was adjusted to $<1 \%$ and minimum score for modified peptides was set $>40$.

\section{Bioinformatics Methods}

Annotation Methods

GO Annotation

The Gene Ontology, or GO, is a major bioinformatics initiative to unify the representation of gene and gene product attributes across all species. More specifically, the project aims to:

1. Maintain and develop its controlled vocabulary of gene and gene product attributes;

2. Annotate genes and gene products, and assimilate and disseminate annotation data;

3. Provide tools for easy access to all aspects of the data provided by the project.

The ontology covers three domains:

1. Cellular component: A cellular component is just that, a component of a cell, but with the proviso that it is part of some larger object; this may be an anatomical structure (e.g. rough endoplasmic reticulum or nucleus) or a gene product group (e.g. ribosome, proteasome or a protein dimer).

2. Molecular function: Molecular function describes activities, such as catalytic or binding activities, that 
occur at the molecular level. GO molecular function terms represent activities rather than the entities (molecules or complexes) that perform the actions, and do not specify where or when, or in what context, the action takes place.

3. Biological process: A biological process is series of events accomplished by one or more ordered assemblies of molecular functions. It can be difficult to distinguish between a biological process and a molecular function, but the general rule is that a process must have more than one distinct steps.

Gene Ontology (GO) annotation proteome was derived from the UniProt-GOA database ( http://www.ebi.ac.uk/GOA/). Firstly, converting identified protein ID to UniProt ID and then mapping to GO IDs by protein ID. If some identified proteins were not annotated by UniProt-GOA database, the InterProScan soft would be used to annotated protein's GO functional based on protein sequence alignment method. Then proteins were classified by Gene Ontology annotation based on three categories: biological process, cellular component and molecular function.

\section{Domain Annotation}

A protein domain is a conserved part of a given protein sequence and structure that can evolve, function and exist independently of the rest of the protein chain. Each domain forms a compact three-dimensional structure and often can be independently stable and folded. Many proteins consist of several structural domains. One domain may appear in a variety of differentially expressed proteins. Molecular evolution uses domains as building blocks and these may be recombined in different arrangements to create proteins with different functions. Domains vary in length from between about 25 amino acids up to 500 amino acids in length. The shortest domains such as zinc fingers are stabilized by metal ions or disulfide bridges. Domains often form functional units, such as the calcium-binding EF hand domain of calmodulin. Because they are independently stable, domains can be "swapped" by genetic engineering between one protein and another to make chimeric proteins.

Identified proteins domain functional description were annotated by InterProScan (a sequence analysis application) based on protein sequence alignment method, and the InterPro domain database was used. InterPro (http://www.ebi.ac.uk/interpro/) is a database that integrates diverse information about protein families, domains and functional sites, and makes it freely available to the public via Web-based interfaces and services. Central to the database are diagnostic models, known as signatures, against which protein sequences can be searched to determine their potential function. InterPro has utility in the large- 
scale analysis of whole genomes and meta-genomes, as well as in characterizing individual protein sequences.

\section{KEGG Pathway Annotation}

KEGG connects known information on molecular interaction networks, such as pathways and complexes (the "Pathway" database), information about genes and proteins generated by genome projects (including the gene database) and information about biochemical compounds and reactions (including compound and reaction databases). These databases are different networks, known as the "protein network", and the "chemical universe" respectively. There are efforts in progress to add to the knowledge of KEGG, including information regarding ortholog clusters in the KEGG Orthology database. KEGG Pathways mainly including: Metabolism, Genetic Information Processing, Environmental Information Processing, Cellular Processes, Rat Diseases, Drug development. Kyoto Encyclopedia of Genes and Genomes (KEGG) database was used to annotate protein pathway. Firstly, using KEGG online service tools KAAS to annotated protein's KEGG database description. Then mapping the annotation result on the KEGG pathway database using KEGG online service tools KEGG mapper.

\section{Subcellular Localization}

The cells of eukaryotic organisms are elaborately subdivided into functionally distinct membrane bound compartments. Some major constituents of eukaryotic cells are: extracellular space, cytoplasm, nucleus, mitochondria, Golgi apparatus, endoplasmic reticulum (ER), peroxisome, vacuoles, cytoskeleton, nucleoplasm, nucleolus, nuclear matrix and ribosomes.

Bacteria also have subcellular localizations that can be separated when the cell is fractionated. The most common localizations referred to include the cytoplasm, the cytoplasmic membrane (also referred to as the inner membrane in Gram-negative bacteria), the cell wall (which is usually thicker in Gram-positive bacteria) and the extracellular environment. Most Gram-negative bacteria also contain an outer membrane and periplasmic space. Unlike eukaryotes, most bacteria contain no membrane-bound organelles, however there are some exceptions.

There, we used wolfpsort a subcellular localization predication soft to predict subcellular localization. Wolfpsort is an updated version of PSORT/PSORT II for the prediction of eukaryotic sequences. Special for protokaryon species, Subcellular localization prediction soft CELLO was used. 
Functional Enrichment

Enrichment of Gene Ontology analysis

Proteins were classified by GO annotation into three categories: biological process, cellular compartment and molecular function. For each category, a two-tailed Fisher's exact test was employed to test the enrichment of the differentially expressed protein against all identified proteins. The GO with a corrected p-value $<0.05$ is considered significant.

Enrichment of pathway analysis

Encyclopedia of Genes and Genomes (KEGG) database was used to identify enriched pathways by a two-tailed Fisher's exact test to test the enrichment of the differentially expressed protein against all identified proteins. The pathway with a corrected $\mathrm{p}$-value $<0.05$ was considered significant. These pathways were classified into hierarchical categories according to the KEGG website.

Enrichment of protein domain analysis

For each category proteins, InterPro (a resource that provides functional analysis of protein sequences by classifying them into families and predicting the presence of domains and important sites) database was researched and a two-tailed Fisher's exact test was employed to test the enrichment of the differentially expressed protein against all identified proteins. Protein domains with a corrected p-value $<0.05$ were considered significant.

\section{Enrichment-based Clustering}

For further hierarchical clustering based on differentially expressed protein functional classification (such as: GO, Domain, Pathway, Complex). We first collated all the categories obtained after enrichment along with their P values, and then filtered for those categories which were at least enriched in one of the clusters with $\mathrm{P}$ value $<0.05$. This filtered $\mathrm{P}$ value matrix was transformed by the function $\mathrm{x}=-\log 10(P$ value). Finally, these $\mathrm{x}$ values were $\mathrm{z}$-transformed for each functional category. These $\mathrm{z}$ scores were then clustered by one-way hierarchical clustering (Euclidean distance, average linkage clustering) in Genesis. Cluster membership were visualized by a heat map using the "heatmap. 2 " function from the "gplotsf" Rpackage. 


\section{Supplementary Figures}

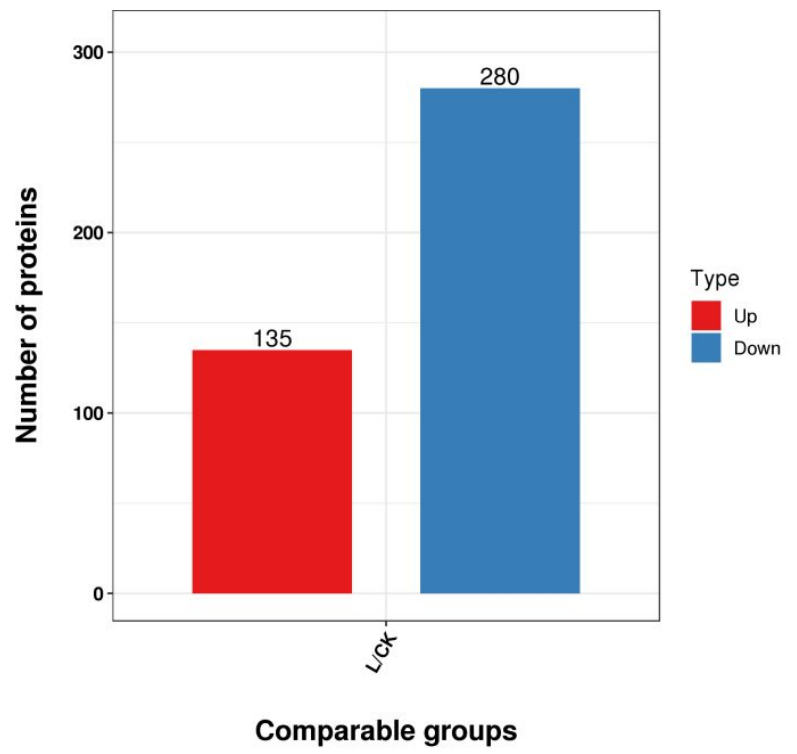

Fig. S1 Histogram of the number distribution of differentially expressed proteins in different comparison groups (L/CK). L represent compound D1. 


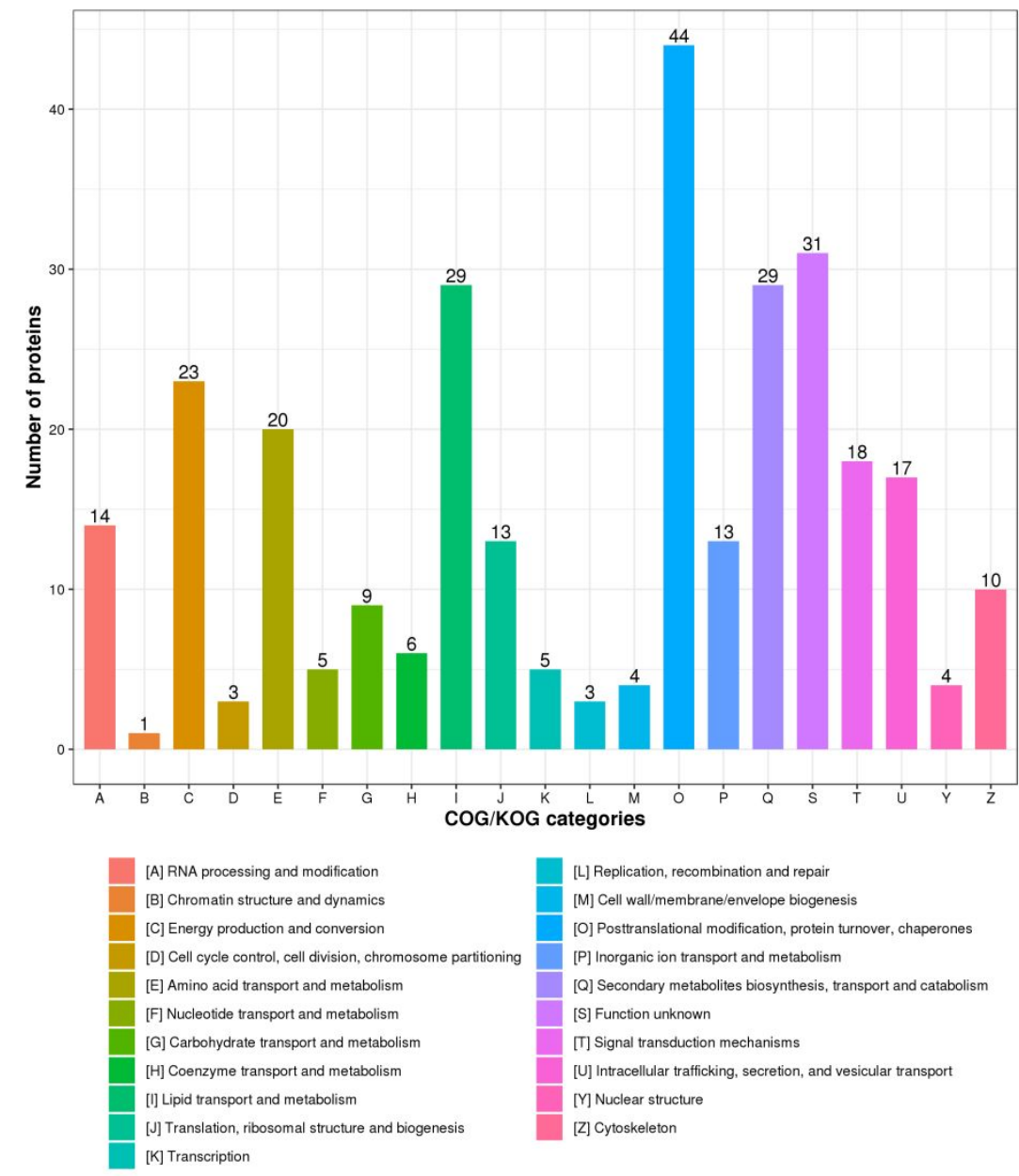

Fig. S2. Functional categorization of proteins with most significantly altered expression in P. capsici exposed to compound D1 according to COG analysis. The numbers in each segment represent the number of proteins allocated to that functional category. 


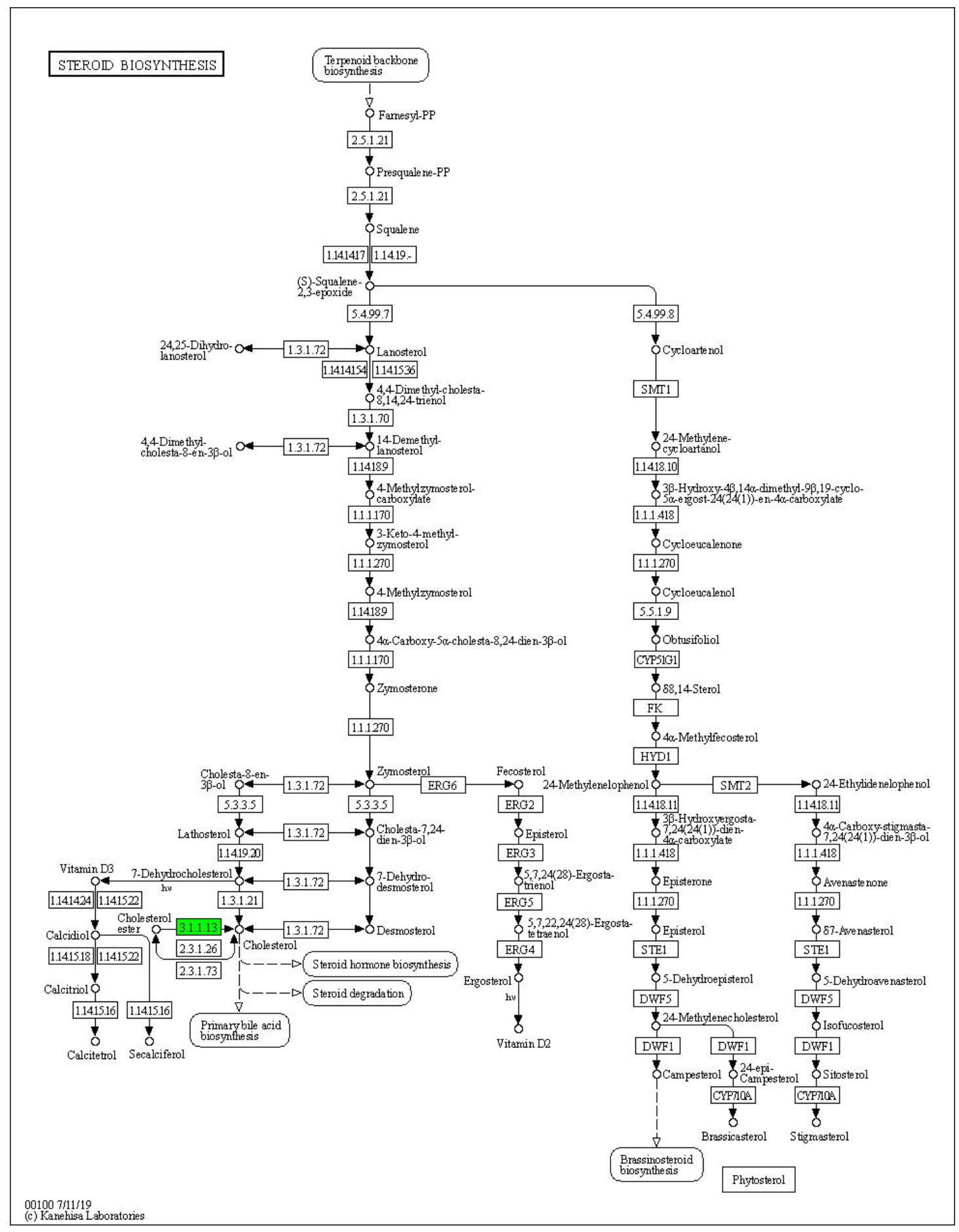

Fig. S3. Metabolism pathways. 


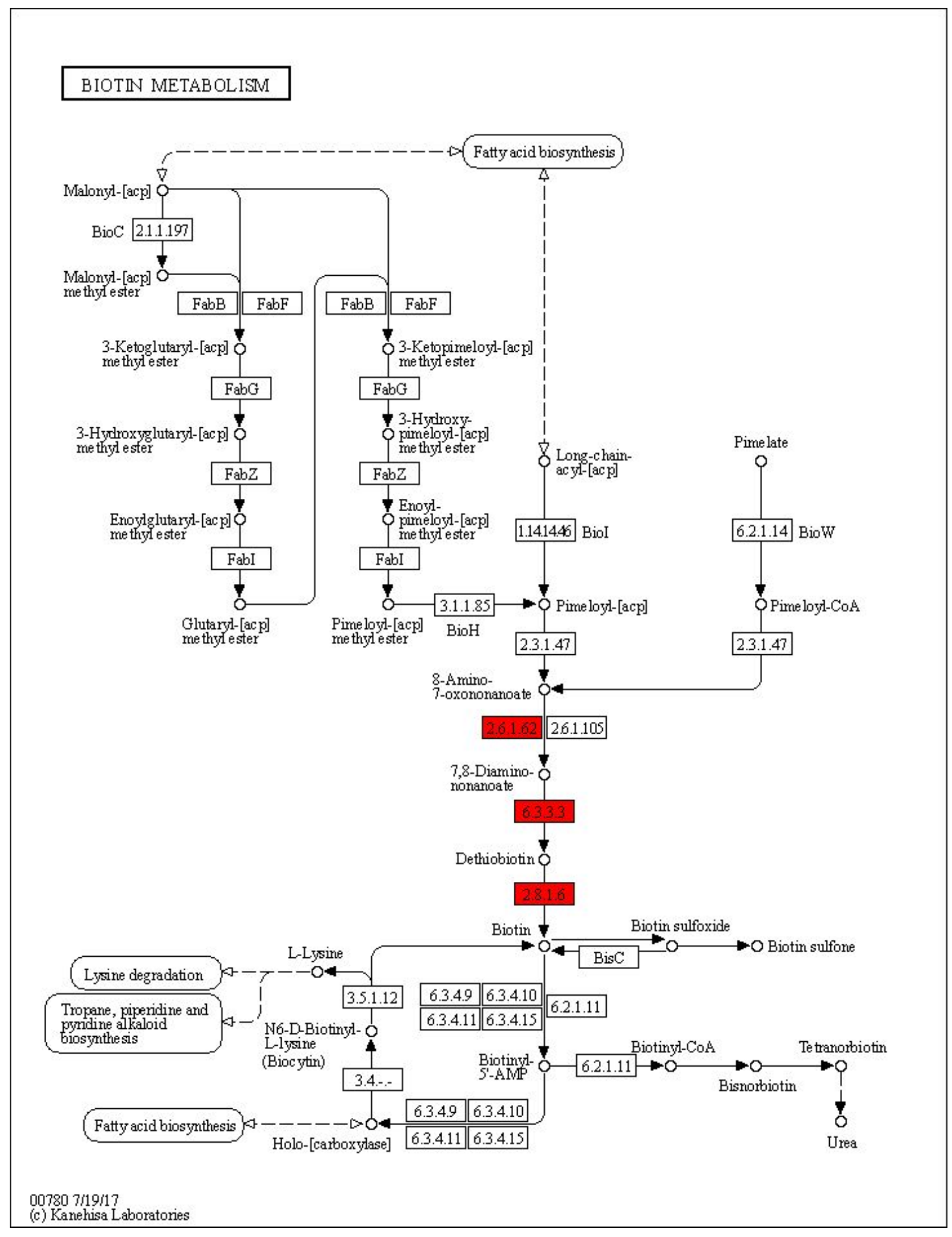




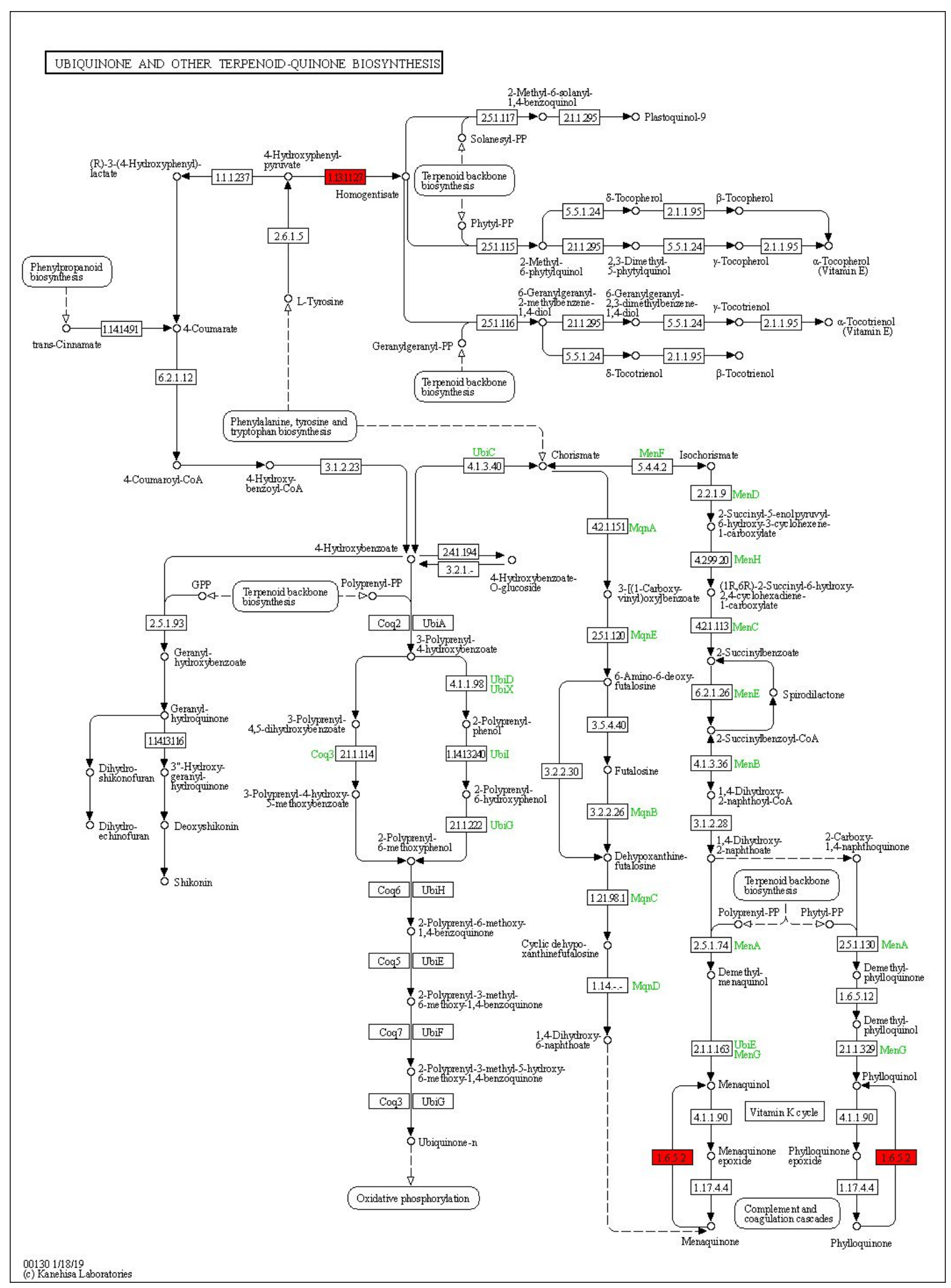



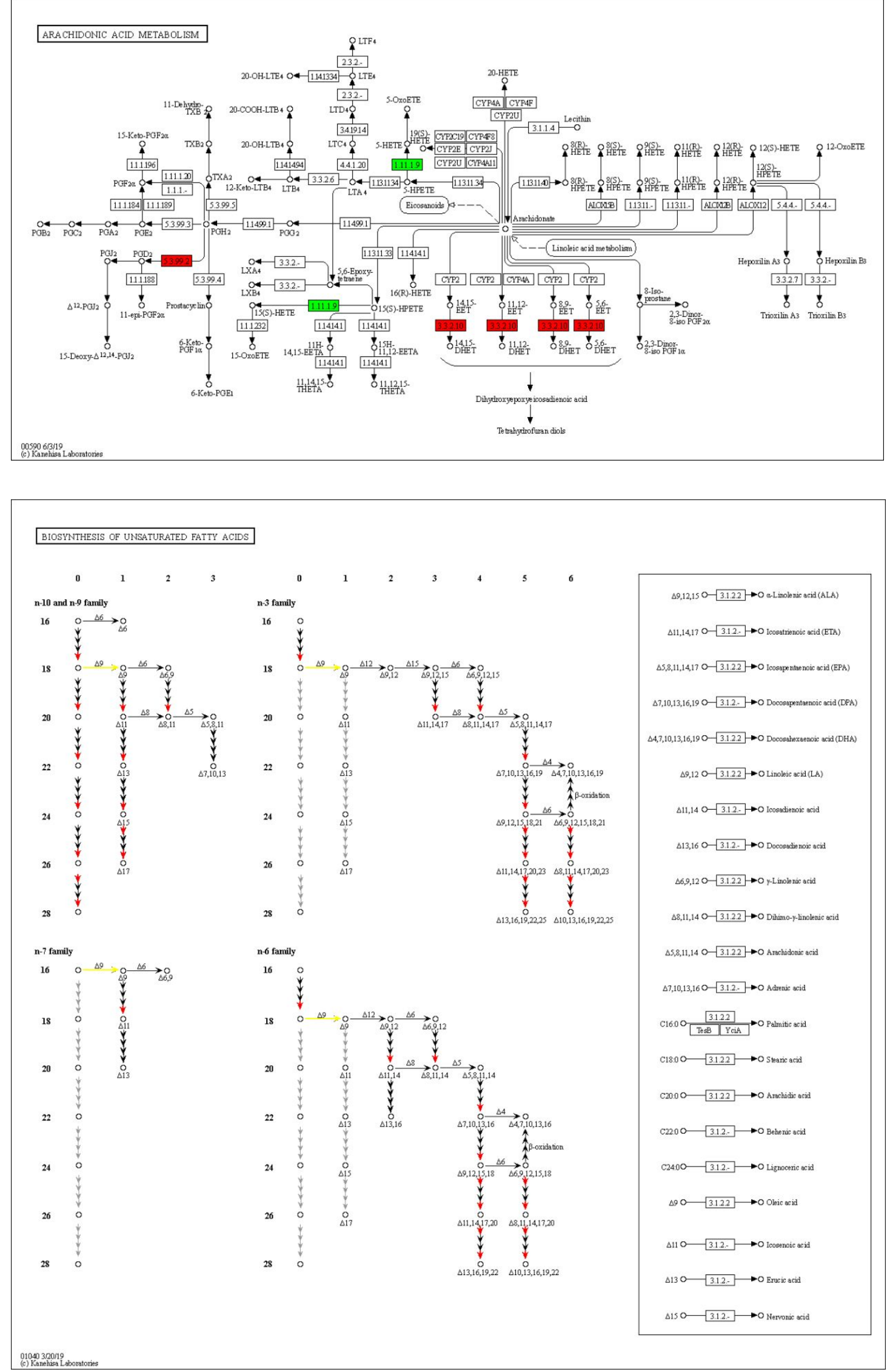


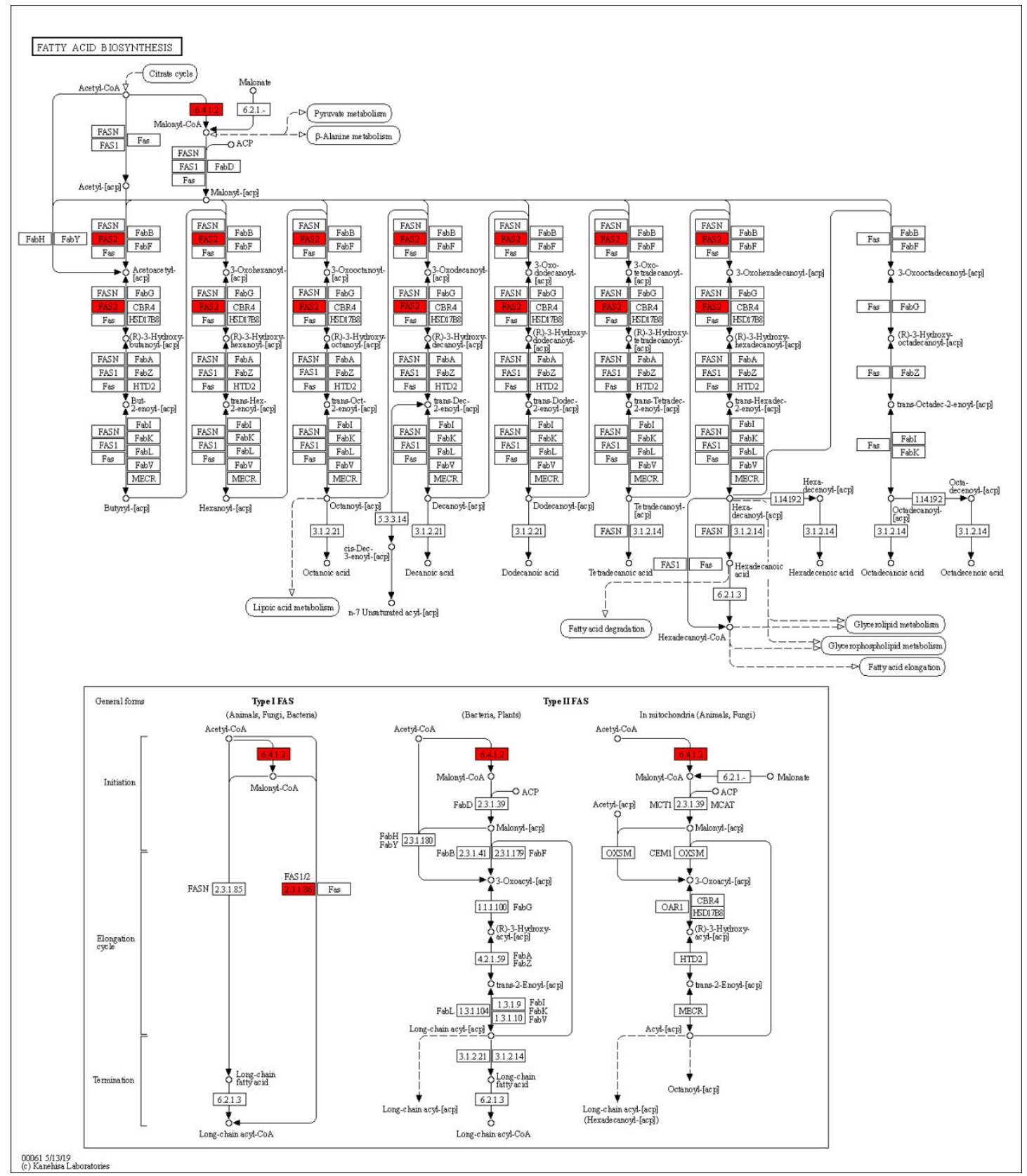


Table S1 Cytotoxic activity of target compounds

\begin{tabular}{ccc}
\hline \multirow{2}{*}{ Entry } & Compd. & In vitro cytotoxicity $\mathrm{IC}_{50}{ }^{a}(\mathrm{\mu g} / \mathrm{mL})$ \\
\cline { 3 - 3 } & No. & HL-7702 \\
\hline 1 & A1 & $>80$ \\
2 & A2 & $78.08 \pm 2.75$ \\
3 & A3 & $53.36 \pm 4.83$ \\
4 & A4 & $52.25 \pm 2.99$ \\
5 & A6 & $>80$ \\
6 & B1 & $23.65 \pm 3.28$ \\
7 & B2 & $>40$ \\
8 & B3 & $30.21 \pm 5.31$ \\
9 & B4 & $35.58 \pm 3.94$ \\
10 & C1 & $>80$ \\
11 & C2 & $>80$ \\
12 & C3 & $>80$ \\
13 & C5 & $>80$ \\
14 & D1 & $>80$ \\
15 & D2 & $59.65 \pm 6.33$ \\
16 & D3 & $>80$ \\
17 & D4 & $>80$ \\
18 & D5 & $>80$ \\
19 & 5-FU & $10.72 \pm 0.91$ \\
\hline
\end{tabular}

${ }^{a} \mathrm{IC}_{50}$ - Compound concentration required to inhibit tumor cell proliferation by $50 \%$.

b Abbreviations: HL-7702 - Human normal liver cells.

c 5 -Fluorouracil, used as a positive control. 


\section{Spectroscopy for target compounds}

All structures of synthesized lactam alkaloids derivatives were confirmed according to their NMR and mass spectroscopy. The typical ${ }^{1} \mathrm{H}$ NMR spectra for target compounds has been presented in the following, which can further confirm the result.

\section{Compd. C6}

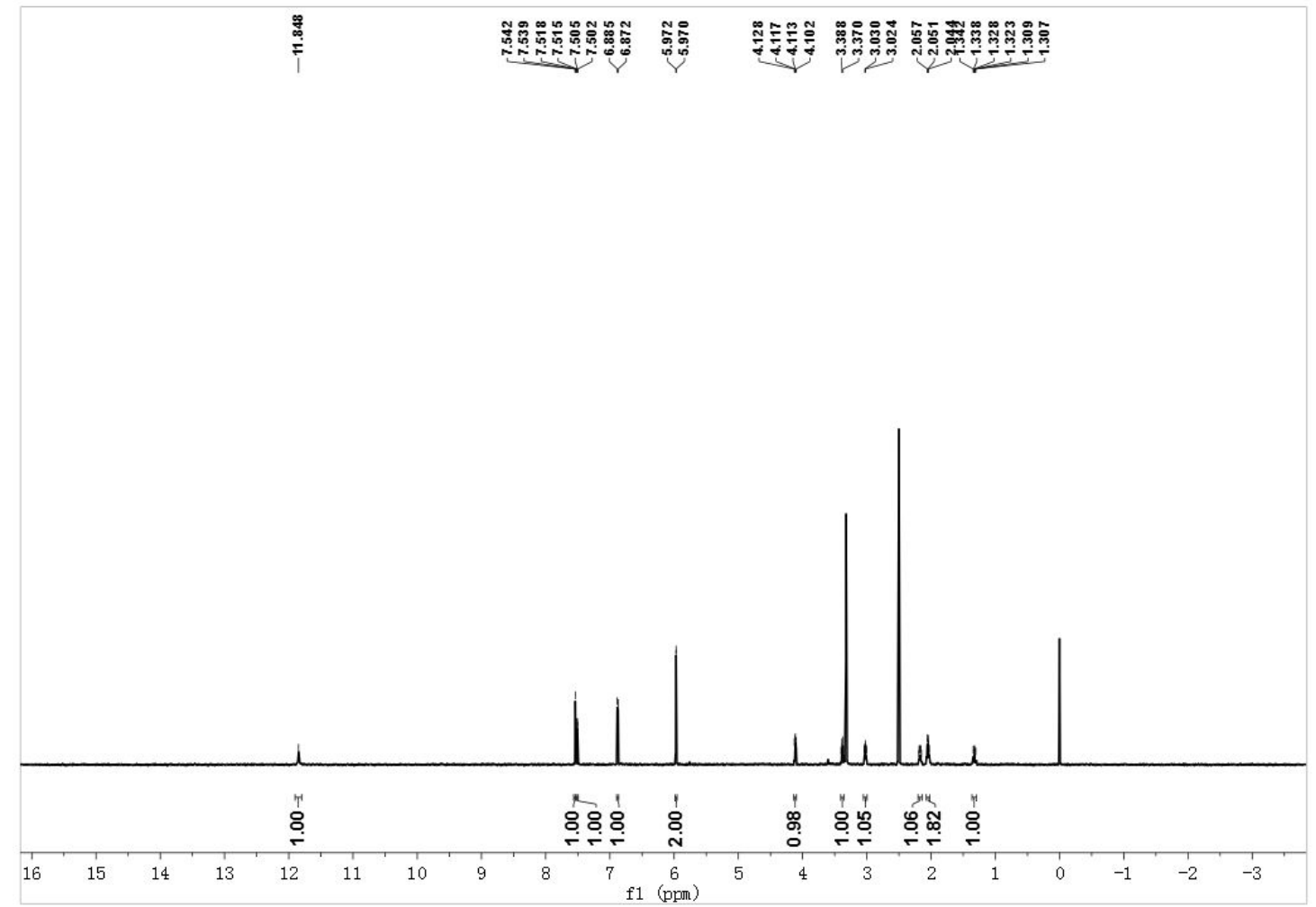

Compd. C7 


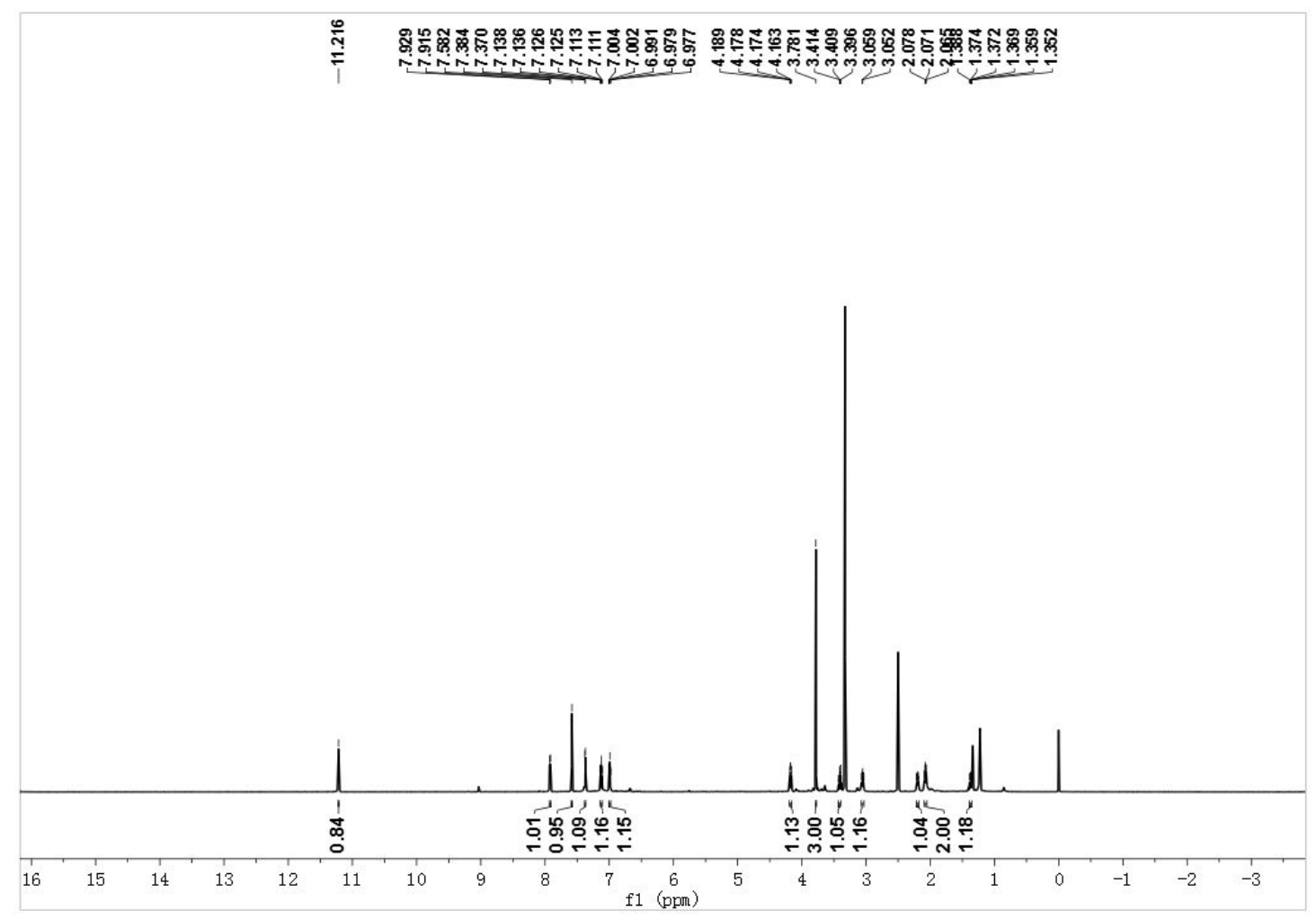

\section{Compd. D1}

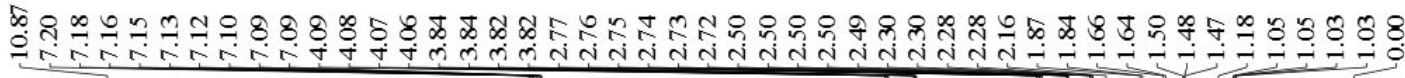

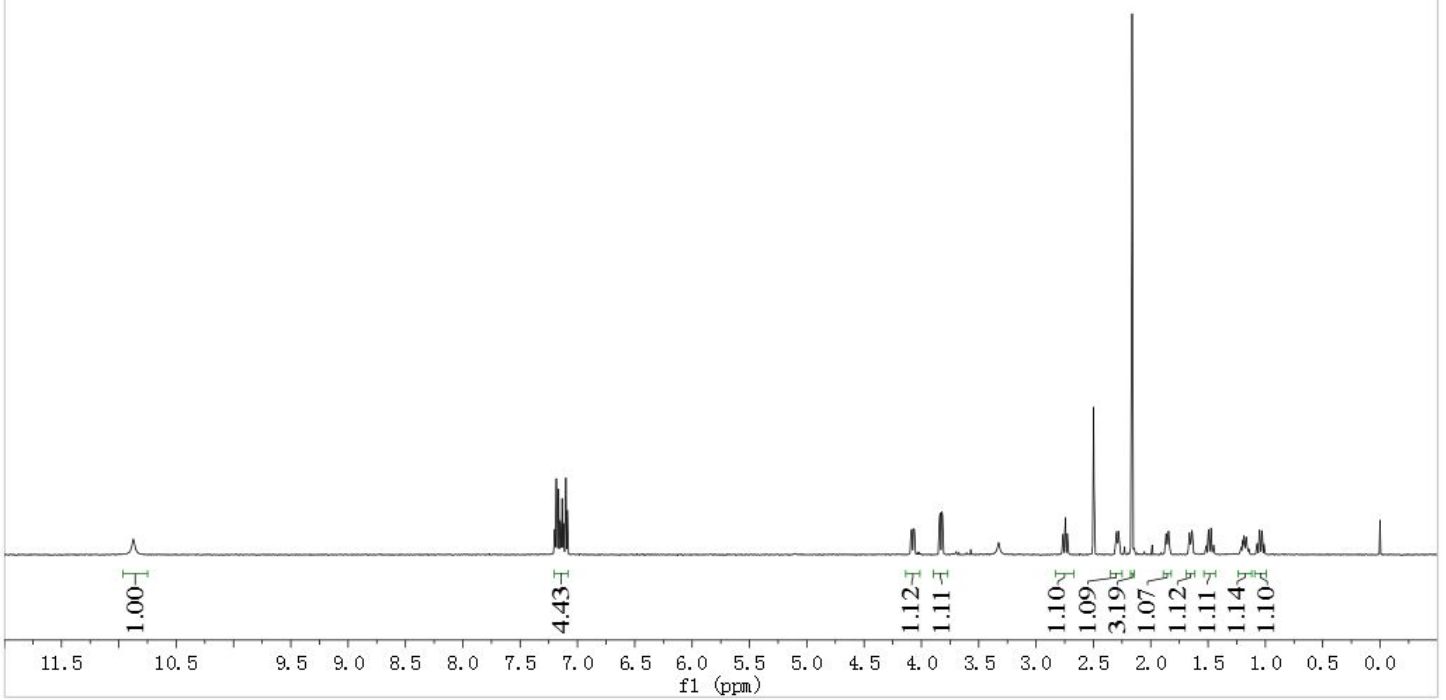

\section{Compd. D2}




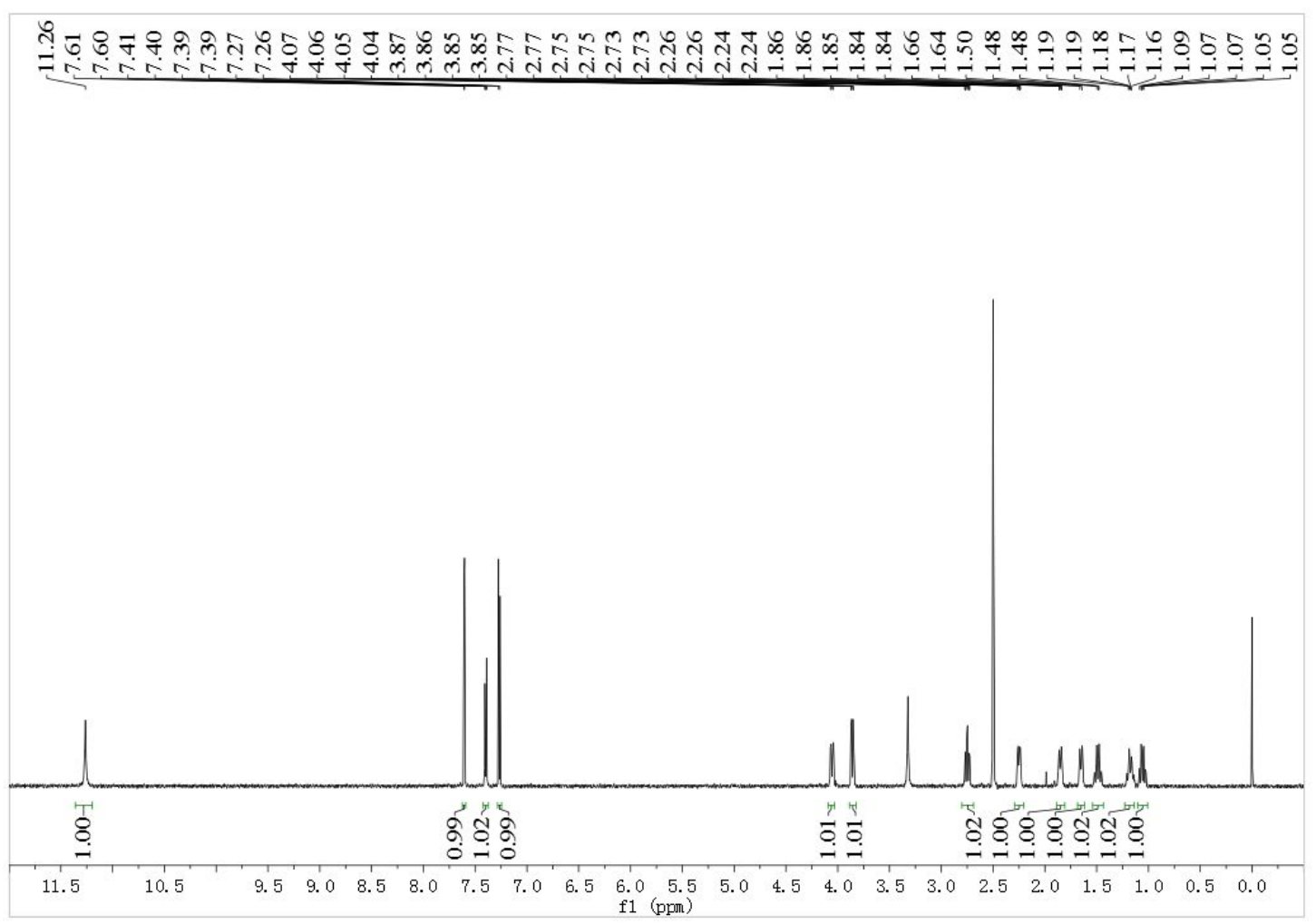

\section{Compd. D3}

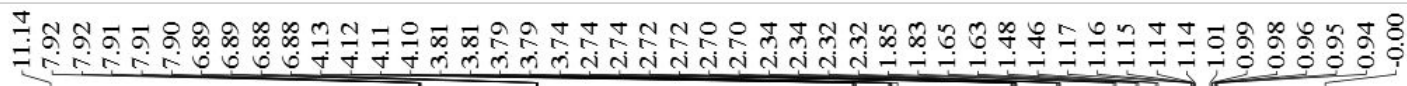

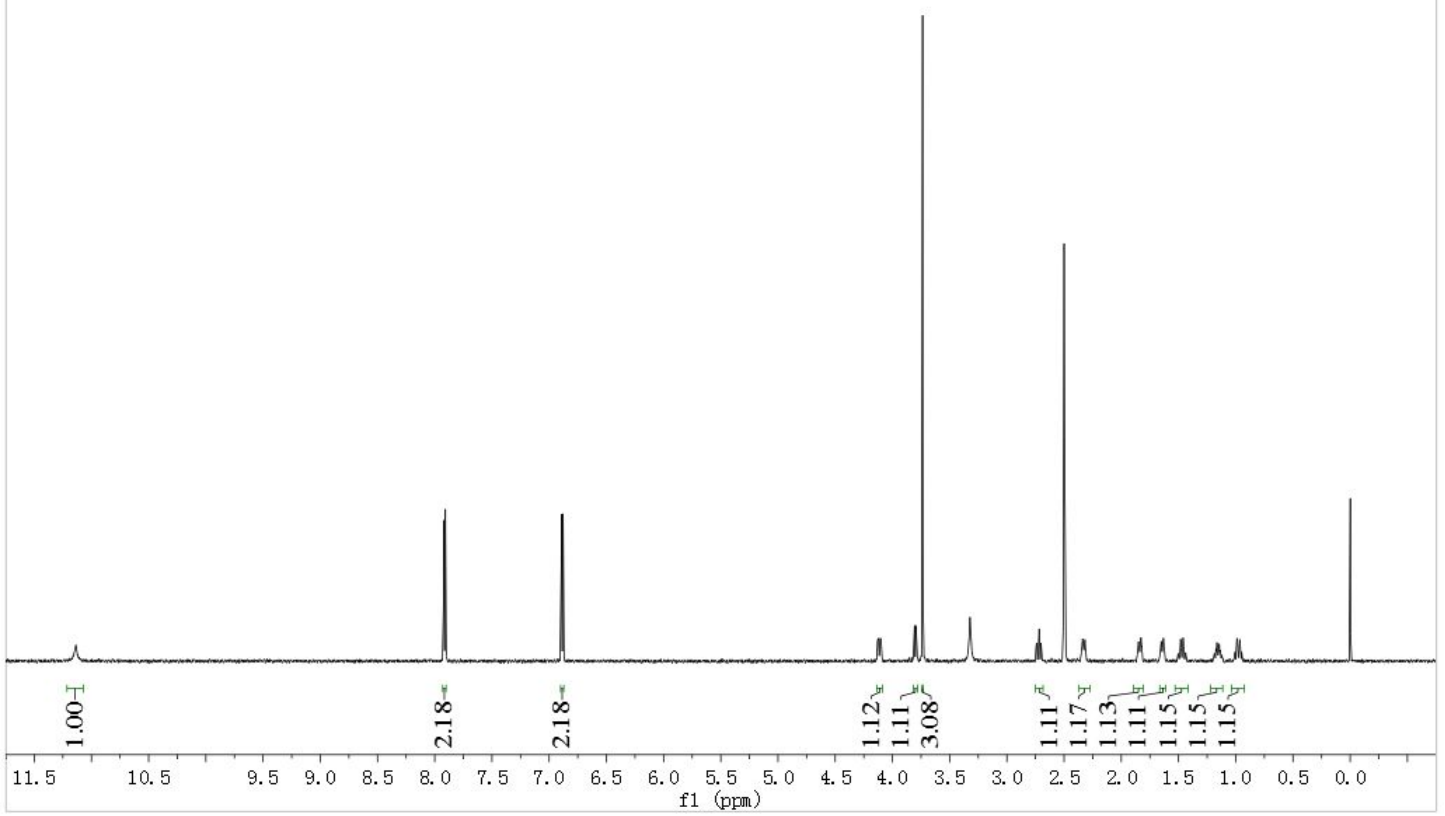

\section{Compd. D4}




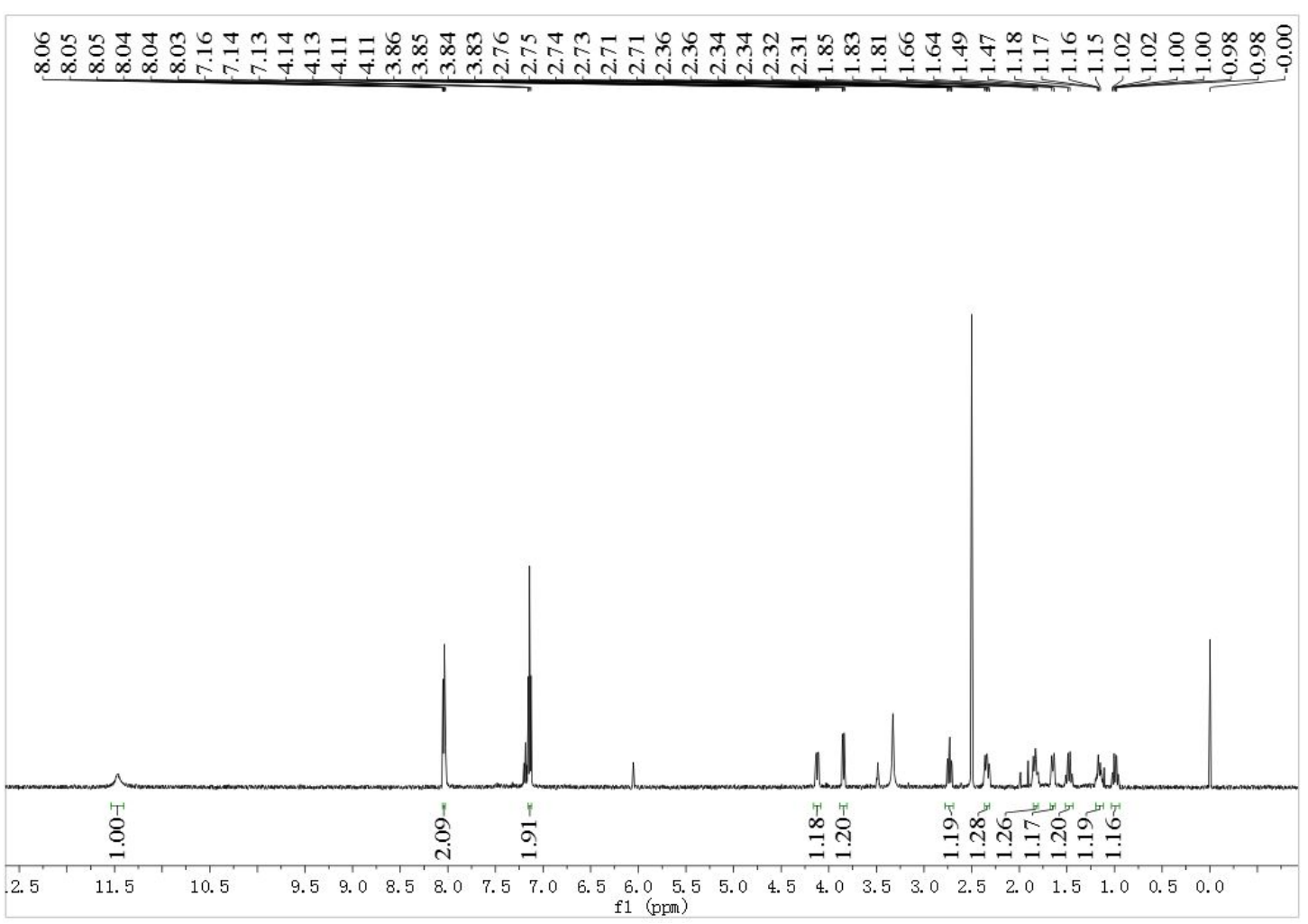

\section{Compd. D5}

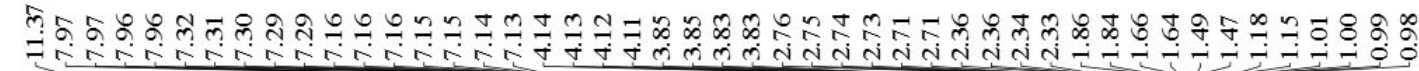

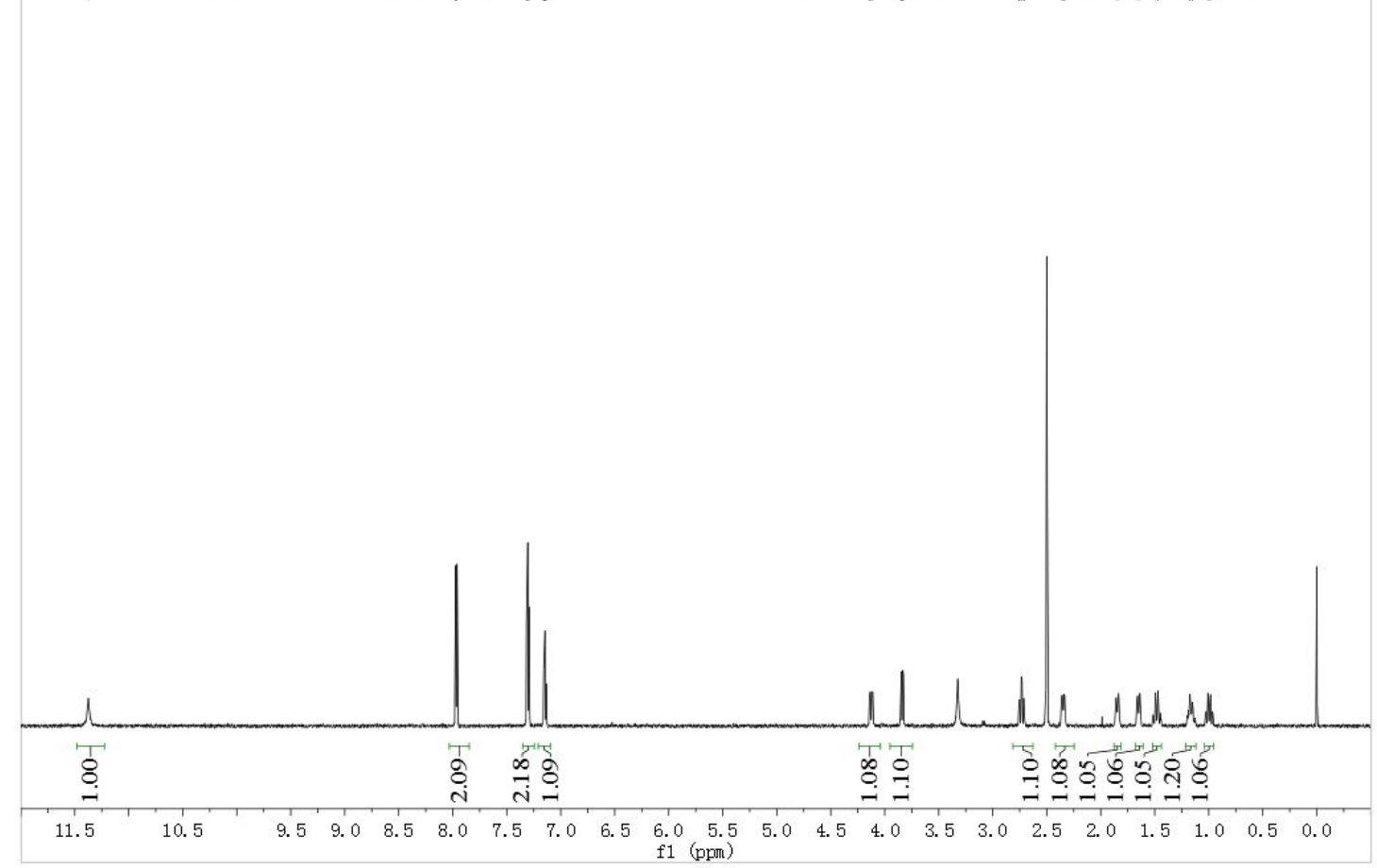

Compd. D6 


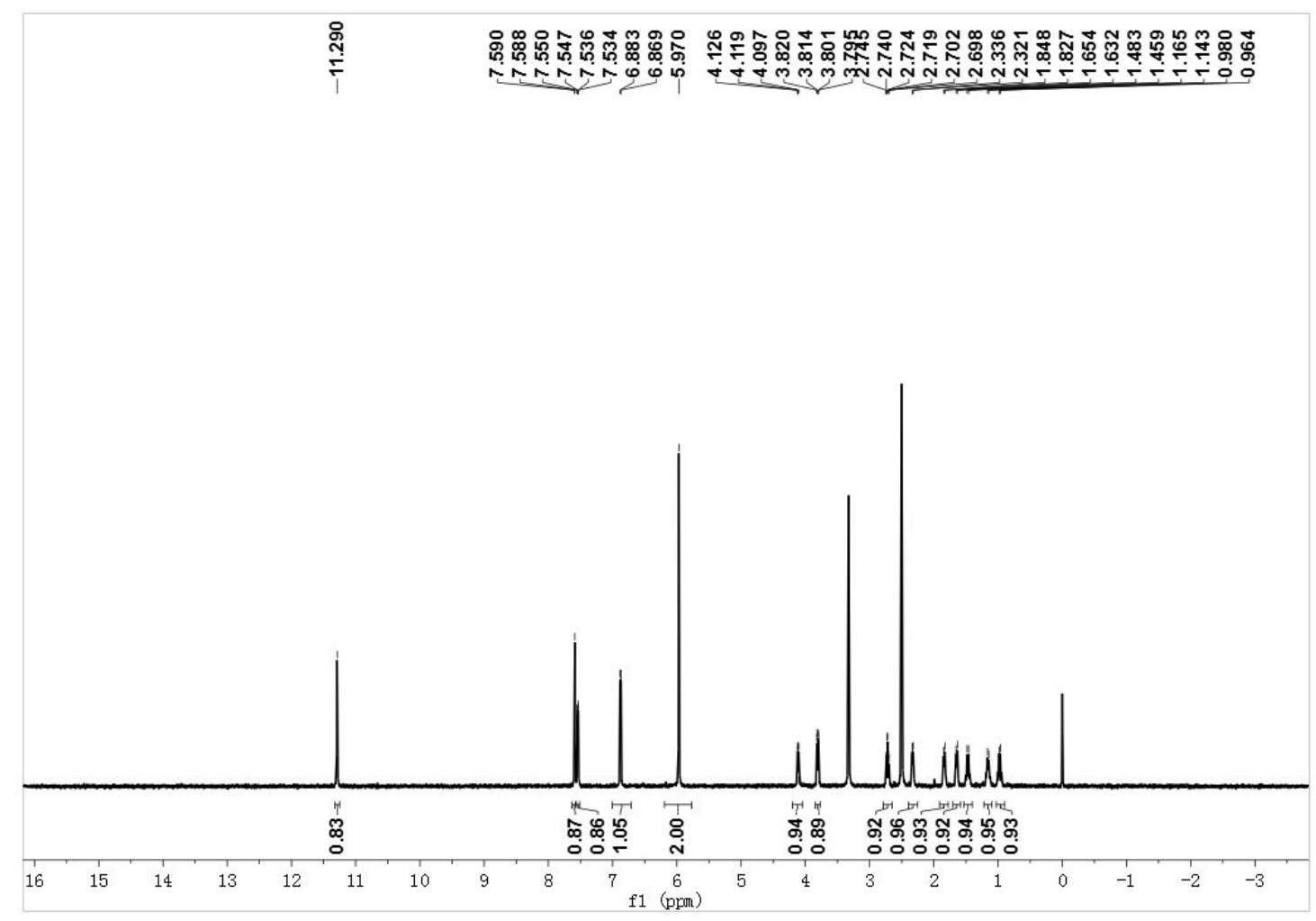

Compd. D7

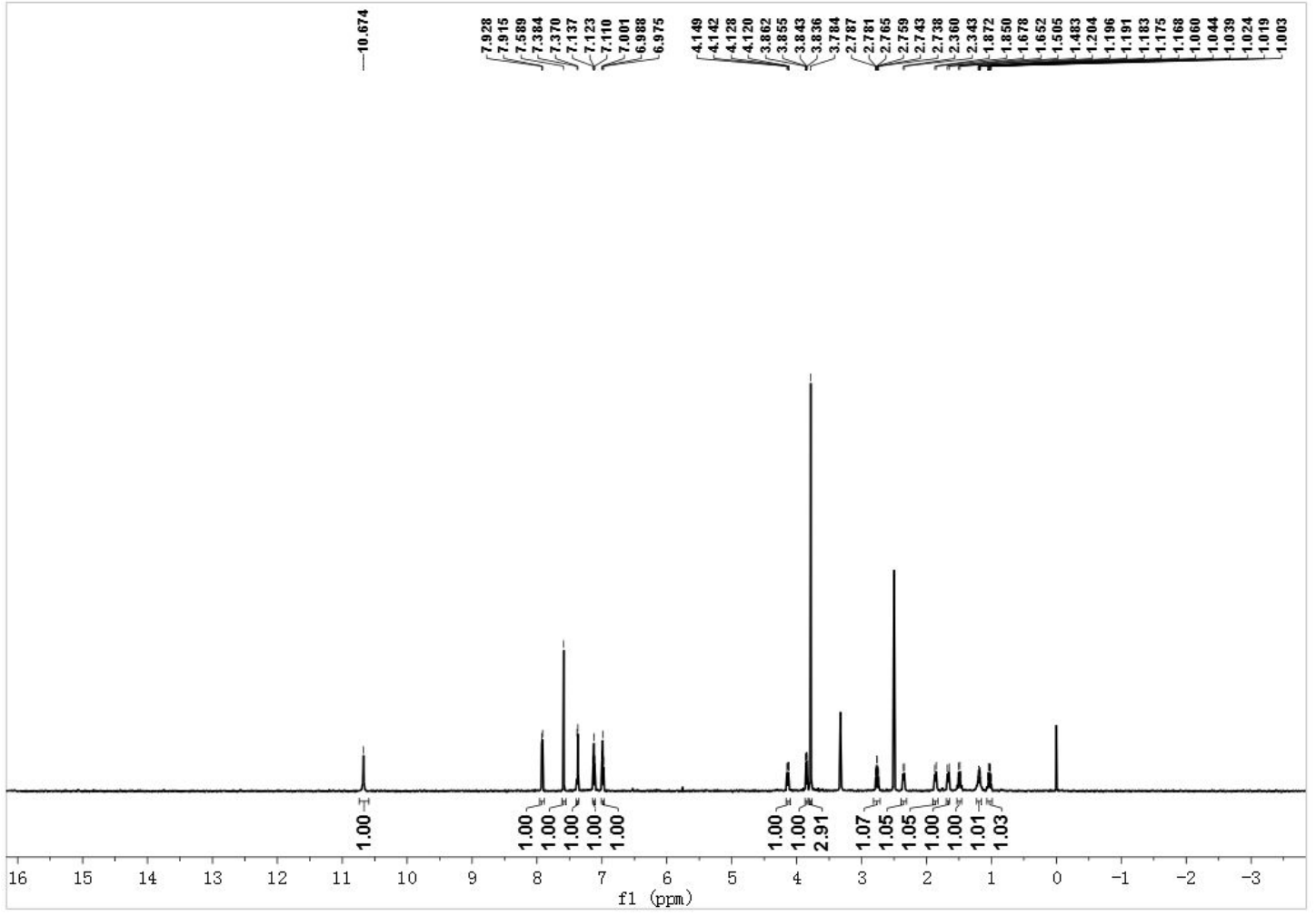

